\title{
Spectral theoretical aspects of anisotropic relativistic models
}

\author{
Balan V. \\ Department Mathematics-Informatics, Politehnica University of Bucharest, Romania; \\ E-mail: Balan<vladimir.balan@upb.ro>;
}

The present work is a survey of results from the spectral theory of covariant symmetric tensors (n-way arrays), which mainly deal with the fundamental geometric objects from anisotropic geometric models recently proposed by Russian specialists in Special Relativity. These objects play a major role in anisotropic structures, being provided by norms and by their related energy scalar fields; in this framework, we study from spectral point of view the m-th root n-way forms, the fundamental metric and the Cartan tensor fields of these models.

The determined spectral data prove to be useful in describing properties of the indicatrices of the anisotropic structures, in pointing out their asymptotic properties and in constructing best rank-I approximations of the main covariant tensors - which provides both simple and consistent estimates for the original anisotropic structures.

Keywords: Finsler structures, n-way arrays, symmetric covariant tensors, $Z$-spectra, $H$-spectra, best rank-I approximation, Cartan tensor, Berwald-Moor metric, $m$-th root structures, fundamental tensor field.

DOI: $10.18698 / 2309-7604-2015-1-67-80$

\section{Spectra associated to $m$-th root relativistic Finsler structures}

The class of $m$-th root Finsler metrics provides for General and Special Relativity (the SRT m-th root models promoted by Pavlov ([28-30]), Chernov ([19]) and Bogoslovsky ([11]), and the Roxburgh spherical symmetric models ([34]), models for ecology ([1]), and extensions for HARDI (Higher Angular Resolution Diffusion Imaging, introduced by L. Astola \& al., [2]). Moreover, some of these metrics alternative non-standard models for Special Relativity, a fruitful subject of research of the last decade.

We shall briefly present first the minimal basics of Finsler structures. Let $(M, F)$ be an $n$-dimensional Finsler or pseudo-Finsler space [27, 11], consisting of a manifold $M$ and a smooth non-negative function (called Finsler norm) $F$ defined on $T M$, which satisfies the following requirements:

a) $F$ is continuous, smooth on the slit tangent space;

b) $F$ is positive homogeneous in the directional argument $\mathrm{y}$, and

c) the halved $y$-Hessian of $F^{2}$, the fundamental metric tensor field is positive-definite.

These assumptions may still be relaxed, by dropping the positivity, the extent of the domain, and by replacing the positive-definiteness with the non-degeneracy and stable signature. Let further 


$$
g_{i j}=\frac{1}{2} \frac{\partial^{2} F^{2}}{\partial y^{i} \partial y^{j}}
$$

be the components of the Finsler metric tensor and let

$$
C_{i j k}=\frac{1}{4} \frac{\partial^{3} F^{2}}{\partial y^{i} \partial y^{j} \partial y^{k}}
$$

be the coefficients of the Cartan symmetric tensor. Due to the positive 1-homogeneity of $F$, by using the Euler relations, one has:

$$
C_{i j k} y^{k}=0 ; g_{i j} y^{j}=F \frac{\partial F}{\partial y^{i}}
$$

It is known that the role of the Cartan tensor is important for identifying the particular structure of a Lagrange space $(M, L)$, since for

$$
C_{i j k}=\frac{1}{4} \frac{\partial^{3} L}{\partial y^{i} \partial y^{j} \partial y^{k}}
$$

completely symmetric and satisfying the property of null y-1-index transvection, the space becomes Finsler, and for a Finsler space with $C_{i j k}=0$, the space is Riemannian (or, in the case of pseudo-Finsler spaces - pseudo-Riemannian).

In the following we mainly describe the spectral data of three types of symmetric tensors:

the Cartan tensor for the Berwald-Moor structure

the metric tensor of the Berwald-Moor structure

in brief, the five attached symmetric tensors related to the Berwald-Moor or, Chernov and Bogoslovsky locally Minkowski Finsler metrics of m-th root type, obtained by polarization (recently described in detail in [3]),

a) the 4-d Berwald-Moor $m$-th root norm and associated tensor 


$$
\begin{aligned}
& F_{B M_{4}}(y)=\sqrt[4]{\left|y_{1} y_{2} y_{3} y_{4}\right|}, \\
& \mathrm{A}_{i j k l}=\frac{1}{4 !}, \text { for }\{i, j, k, I\}=\{1,2,3,4\}
\end{aligned}
$$

b) the 3-d Berwald-Moor $m$-th root norm and associated tensor:

$$
\begin{aligned}
& F_{B M_{3}}(y)=\sqrt[3]{\left|y_{1} y_{2} y_{3}\right|}, \\
& \mathrm{A}_{i j k l}=\frac{1}{3 !}, \text { for }\{i, j, k\}=\{1,2,3\}, 0-\text { otherwise }
\end{aligned}
$$

b) the 4-d Chernov $m$-th root norm and associated tensor:

$$
\begin{gathered}
F_{C_{3}}(y)=\sqrt[3]{\left|y_{1} y_{2} y_{3}+y_{1} y_{2} y_{4}+y_{1} y_{3} y_{4}+y_{2} y_{3} y_{4}\right|}, \\
B_{i j k}=\frac{1}{3 !}, \text { for distinct }\{i, j, k\} \subset\{1,2,3,4\}
\end{gathered}
$$

c) the 3-d Chernov $m$-th root norm and associated tensor (Minkowski-Lorentz framework):

$$
\begin{gathered}
F_{C_{3}}(y)=\sqrt[3]{\left|y_{1} y_{2}+y_{1} y_{3}+y_{2} y_{3}\right|}, \\
B_{i j k}=\frac{1}{3 !}, \text { for distinct }\{i, j\} \subset\{1,2,3\}
\end{gathered}
$$

d) the 3-d Bogoslovsky $m$-th root norm and associated tensor:

$$
\begin{aligned}
& F_{B_{3}}(y)=\sqrt[4]{\left|y_{1}{ }^{2} y_{2} y_{3}+y_{2}{ }^{2} y_{3} y_{1}+y_{3}{ }^{2} y_{1} y_{2}\right|}, \\
& \mathrm{B}_{i j k l}=\frac{1}{36}, \text { for }\{i, j, k, l\}=\{1,2,3\}
\end{aligned}
$$

\section{Basics on the spectral theory of covariant symmetric tensors ([3])}

Consider a real $m$-covariant symmetric tensor field $T$ on the flat manifold $V=\boldsymbol{R}^{n}$. We say that a real $\lambda$ a $Z$-eigenvalue that a vector $y$ is an associated $Z$-eigenvector to $\lambda$, if they satisfy the 
system:

$$
T_{y}^{m-1}=\lambda y ; g(y, y)=1
$$

where we denoted

$$
T_{y}^{m-1}=\sum_{i, i_{2}, \ldots, i_{m} \in 1, n} \mathrm{~T}_{i i_{2} \ldots i_{m}} y_{i_{2}} \ldots y_{i_{m}} \mathrm{dx}^{i}
$$

In the complex case, one simply calls $\lambda$ and $y$ eigenvalue and eigenvector, respectively.

L. Qi defined the following alternative for spectral objects:

A real number $\lambda$ is an $H$-eigenvalue and a vector $y$ is an $H$-eigenvector associated to $\lambda$, if they satisfy the homogeneous polynomial system of order $m-1$ :

$$
\left(T_{y}^{m-1}\right)_{k}=\lambda\left(y_{k}\right)^{m-1}
$$

In the complex case, $\lambda$ and y are called $E$-eigenvalue and $E$-eigenvector, respectively.

Regarding the spectra consistency, it is known that the $Z$ - and the $H$-spectra are nonempty for even symmetric tensors, and that a symmetric tensor $T$ is positive definite/semi-definite iff all its $H$ - (or $Z$-) eigenvalues are positive/non-negative.

In general, while considering an $m$-multilinear symmetric form $T$ defined on $V$, we note that the definition of $Z$ - and $H$-spectral data reveal certain relations between the poly-angles determined by the poly-scalar product $T$ and the classic Euclidean and Riemann-Finsler geometric structures, as follows.

a) Denoting by $\delta$ the Euclidean inner product, the $Z$-eigensystem for $\lambda$ and y can be written as:

$$
\left(T_{y}^{m-1}, \mathrm{z}\right)=\lambda \delta(y, z), \forall z \in \mathbb{R}^{n},\|y\|_{2}=1
$$

i.e., the (m-1)-polyangle determined by the poly-scalar product $T$ and the classic Euclidean inner product, based on $Z$-eigenvectors of $T$, are homothetic while applied to Euclidean unit vectors.

b) Denoting by $C$ the Riemann-Finsler multilinear symmetric form associated to the $m$-pseudonorm 


$$
F_{R F}(y)=\sqrt[m]{y_{1}^{m}+\ldots+y_{n}^{m}}
$$

namely

$$
C=\sum_{i=1}^{n} \otimes^{m} d x^{i}=\sum \delta_{i_{1} \ldots i_{m}} d x^{i_{1}} \otimes \ldots \otimes d x^{i_{m}}
$$

we note that the $H$-eigensystem can be written as:

$$
\left(T_{y}^{m-1}, \mathrm{z}\right)=\lambda C\left(y^{m-1}, z\right), \forall \mathrm{z} \in \mathbb{R}^{n},\|y\|_{2}=1
$$

i.e., the (m-1,1)-polyangles of the poly-scalar products $T$ and $C$, based on the $H$-eigenvectors of $T$, are homothetic for Euclidean unit vectors. The eigenvalues defined by $T$ can be characterized in terms of homothety of linear forms, as follows ([3]):

a) Consider the covector-providing mappings

$$
\delta_{*}, \mathrm{~T}_{*}: \mathcal{T}_{0}^{1}(V) \rightarrow \mathcal{T}_{1}^{0}(V)
$$

given by

$$
\begin{gathered}
\delta_{*}(y)=\sum_{i \in 1, n} y_{i} d x^{i}, \\
T_{*}(y)=\sum_{i, i_{1}, \ldots, i_{m-1} \in \overline{1, n}} T_{i_{1} \ldots i_{m-1}} y_{i_{1} \ldots y_{i_{m-1}}} d x^{i}
\end{gathered}
$$

Then the real scalar $\lambda$ is a $Z$-eigenvalue and the unit-vector $y$ is an associated $Z$-eigenvector iff

$$
T_{*}(y)=\lambda \delta_{*}(y)
$$

i.e., the two defined by $y$ Riesz linear forms attached to $T *$ and $\delta$ are homothetic with factor $\lambda$.

b) The extended $m$-th root of sum of $m$-th powers of components Riemann-Finsler metric provides the associated mapping 


$$
C_{*}: \mathcal{T}_{0}^{1}(V) \rightarrow \mathcal{T}_{1}^{0}(V), C_{*}(y)=\sum_{i \in 1, n}\left(y_{i}\right)^{m-1} d x^{i} .
$$

Then the real scalar $\lambda$ is an $H$-eigenvalue of $T$ with associated $H$-eigenvector $y$ if

$$
T_{*}(y)=\lambda C_{*}(y),
$$

i.e., the two Riesz-type linear forms attached to $T$ and $C$ defined by $y$ are homothetic with factor $\lambda$. Similarly, in the complex case, the last property can be rephrased for $E$-spectra.

We direct the reader for details on the asymptotic rays, recession vectors, the degeneracy sets, the best rank-I approximation and the $Z$ - and $H$-spectral data for the five structural a) - e) to the article [3]. It should still be noted, that while the Berwald-Moor fundamental tensor allows eigensystems with tractable resultants, the eigendata for the Chernov and Bogoslovsky tensors are by far more untractable and complex by structure. E.g., even for $n=3$, in the Chernov ([19]) case, one gets the $Z$-spectral data ([3]):

$$
\begin{aligned}
& \left\{\begin{array}{l}
S_{\lambda=-1}=\left\{\left(\rho_{1}, \rho_{2}, t \mid t \in D=\left[-\sqrt{\frac{2}{3}}, \sqrt{\frac{2}{3}}\right], v=\sqrt{2-3 t^{2}}\right\},\right. \\
S_{\lambda=2}=\{ \pm(1,1,1) / \sqrt{2}\} .
\end{array},\right. \\
& \text { where } \rho_{1}=\frac{-t \pm v}{2} \text { and } \rho_{2}=\frac{-t \mp v}{2}
\end{aligned}
$$

while for the Bogoslovsky tensor, just a partial result shows that the $Z$-spectral equations for $\mathrm{v}=(a, b, c)$ have the form

$$
\left\{\begin{array}{l}
24 a b c+2\left(b^{2} c+c^{2} b\right)=\lambda a, \\
24 a b c+2\left(c^{2} a+a^{2} c\right)=\lambda b, \\
24 a b c+2\left(a^{2} b+b^{2} a\right)=\lambda c, \\
a^{2}+b^{2}+c^{2}=1,
\end{array}\right.
$$


and admit as particular solution $\lambda=28 / 3$ and one generating unit eigenvector $y=(1,1,1) / \sqrt{ } 3$.

We shall further present the solutions for the spectral equations for Berwald-Moor Cartan tensor. Considering the slit tangent bundle of the manifold $\mathrm{M}$ endowed with Euclidean structure given by the flat metric the spectral $Z$ - and $H$ - $/ E$ - equations for the Cartan tensor $C$ respectively have the form

$$
C_{i j k} f^{j} f^{k}=\lambda f^{i}, \text { with }\|f\|_{2}=1 ; \quad C_{i j k} f^{j} f^{k}=\lambda\left(f^{i}\right)^{2},
$$

where is a local vertical vector field. In particular, in the Berwald-Moor $n$-dimensional case we have (1978, Matsumoto-Shimada)

$$
\begin{aligned}
& g_{i j}=\frac{F^{2}}{n y^{i} y^{j}}\left(\frac{2}{n}-\delta_{i j}\right) \\
& C_{i j k}=\frac{F^{2}}{n y^{i} y^{j} y^{k}}\left(\frac{2}{n^{2}}-\frac{\delta_{i j}+\delta_{j k}+\delta_{k i}}{n}+\delta_{i k} \delta_{j k} \delta_{k i}\right) .
\end{aligned}
$$

By denoting

$$
g^{i}=f^{i} / y^{i}, g^{*}=\sum_{k=1}^{n} g^{k} \text { and } g^{\#}=\sum_{k=1}^{n}\left(g^{k}\right)^{2}
$$

the $Z$-spectral equations for the metric $g$ of the Berwald-Moor space have the form ([4]):

$$
\frac{F^{2}}{n^{3} y^{i}}\left[2\left(g^{*}\right)^{2}-2 n g^{*} g^{i}-n g^{\#} n^{2}\left(g^{i}\right)^{2}\right]=\lambda\left(g^{i} g^{i}\right)^{k}, i=\overline{1, n},
$$

with $\kappa=1$ for $Z$-spectrum and $\kappa=2$ for $\mathrm{H}$-/E-spectra. In particular, for $n=2$ the Z-equations are much simpler,

$$
0=\lambda\left(g^{i} g^{i}\right)^{k}, i=\overline{1, n},
$$

the space is pseudo-Euclidean of Minkowski type and hence has vanishing Cartan tensor. Then the only $Z$ - and $H$ - $/ E$-eigenvalue of the Cartan tensor is the null one. Further, for $n=3$ and denoting $(a, b, c)=\left(g^{1}, g^{2}, g^{3}\right)$, , the system of three $Z$ - and $H$-/E-equations have respectively the form: 


$$
\begin{aligned}
& \mathcal{R}:\left[3 \mathrm{a}^{2}+6 b c-(a+b+c)^{2}\right]=27 \lambda a \sqrt[3]{\frac{\left(y^{1}\right)^{4}}{\left(y^{2} y^{3}\right)^{2}}} \\
& \mathcal{R}:\left[3 \mathrm{a}^{2}+6 b c-(a+b+c)^{2}\right]=27 \lambda a^{2} \sqrt[3]{\frac{\left(y^{1}\right)^{7}}{\left(y^{2} y^{3}\right)^{2}}}
\end{aligned}
$$

where by $R$ we denote the simultaneous rolling of the triples $(a, b, c)$ and $(1,2,3)$.

For $n=4$, by denoting $(a, b, c, d)=\left(g^{1}, g^{2}, g^{3}, g^{4}\right)$, the systems of four equations have the form:

$$
\begin{aligned}
& \mathcal{R}:\left[-2(\mathrm{a}+\mathrm{b}+\mathrm{c}+\mathrm{d})^{2}+8\left(a^{2}+b c+c d+d b\right)\right]= \\
& =64 \lambda a \sqrt{\frac{\left(y^{1}\right)^{3}}{\left(y^{2} y^{3} y^{4}\right)^{2}}}, \\
& \mathcal{R}:\left[-2(\mathrm{a}+\mathrm{b}+\mathrm{c}+\mathrm{d})^{2}+8\left(a^{2}+b c+c d+d b\right)\right]= \\
& =64 \lambda a^{2} \sqrt{\frac{\left(y^{1}\right)^{5}}{\left(y^{2} y^{3} y^{4}\right)^{2}}},
\end{aligned}
$$

where by $R$ we denote the simultaneous rolling of the triples $(a, b, c, d)$ and $(1,2,3,4)$.

Remarks. We note that, due to the properties of the Finslerian Cartan tensor, all spectra admit the real eigenvalue zero, with the associated family of $Z$-eigenvectors containing the two unit vectors attached to the supporting element $y$, and the family of $H$-eigenvectors containing the eigenspace $\operatorname{Span}\{y\}$. The complexity of computations, which address the Theory of Resultants related to higher-order polynomial systems of equations, often require support or at least accurate validation by means of symbolic software. Using the Maple 15 environment, the spectral $\mathrm{N}$-way theory is tractable for our cases ([4]), as described in the following.

For the Berwald-Moor 3-d case, there exist points at which the Cartan tensor admits a Candecomp/Parafac best rank-I approximation (relative to the Euclidean-originated Frobenius norm). Namely, in each Euclidean fiber at $(x, y)$-with $\|y\|=1 /(\lambda \sqrt{3})$ on the trisectrix, the $Z$-spectral data is given by:

$$
S_{\lambda=0}^{Z}=\{(1,1,1) / \sqrt{3}\},
$$




$$
\left.S_{\lambda= \pm 1 /(3 \sqrt{2} \mid y \|)}^{Z}=\{(2,1,1) / 3 \sqrt{6} \lambda)\right\}^{\sigma}
$$

denotes complementing the set with the vectors obtained by complete permuting the components. In this case, the Cartan tensor admits six distinct Candecomp (best rank-I) approximations:

$$
C^{\sigma} m \frac{1}{\|y\|} f_{b} \otimes f_{b} \otimes f_{b}
$$

For $n=3$, there exist points at which the Cartan tensor admits only purely complex HO-SVD decomposition. Namely, in each Euclidean fiber at $(x, y)$ - with $y=(1,1,1)$ on the trisectrix, there exists only one $H$-eigenvalue $(\lambda=0)$ with all $H$-eigenvectors located along the trisectrix, three distinct $E$-eigenvalues (one real and 2 complex conjugate), each allowing three complex 1dimensional eigenspaces. We have

$$
\begin{aligned}
& S_{\lambda=0}^{H}=\operatorname{Span}\{(1,1,1)\} \\
& S_{\lambda=1 / 3}^{E}=\operatorname{Span}\{((-1 \pm i \sqrt{3}) / 2,(-1 \pm \mathrm{i} \sqrt{3}) / 2,1)\}^{\sigma} \\
& S_{\lambda=(1 \pm 2 i \sqrt{2}) / 27}^{E}=\operatorname{Span}\{( \pm i \sqrt{2}, 1,1)\}^{\sigma} .
\end{aligned}
$$

For $n=4$, there exist points at which the Cartan tensor admits a Candecomp/Parafac best rank-I approximation (relative to the Euclidean-originated Frobenius norm). Namely, in each Euclidean fiber at $(x, y)$-with $y$ on the quadrisectrix, we have:

$$
\begin{gathered}
S_{\lambda=0}^{Z}=\{ \pm(1,1,1,1) / 2\}, \\
\left.S_{\lambda= \pm 1 /(2 \sqrt{3}|y| \mid)}^{Z}=\{ \pm(3,-1,-1,-1) / 2 \sqrt{3})\right\}^{\sigma}
\end{gathered}
$$

As well, for $n=4$, there exist points at which the Cartan tensor admits only a purely complex HO-SVD decomposition. The $H$-/E-spectral data for the Cartan tensor in the fiber at $(x, y)$-with $y=(1,1,1,1)$ is given by: 


$$
\begin{aligned}
& S_{\lambda=0}^{H}=\operatorname{Span}\{(1, r, r, 1)\}^{\sigma}, r \in \mathbb{R} ; \\
& S_{\lambda=1 / 4}^{E}=\operatorname{Span}\left\{(1, \bar{\tau}, \tau, 1\}^{\sigma} \cup \operatorname{Span}\{(\rho, \bar{\rho}, \bar{\rho}, 1)\}^{\sigma} \cup\right. \\
& \cup \operatorname{Span}\{-1, \theta, \bar{\theta}, 1)\}^{\sigma} \cup \operatorname{Span}\{(\alpha, q,-\alpha, 1)\}^{\sigma} \\
& S_{\lambda=(1 \pm i \sqrt{3}) / 16}^{E}=\operatorname{Span}\{( \pm i \sqrt{3}, 1,1,1)\}^{\sigma}, \\
& \text { where } \alpha=\left[-(q+1) \pm \sqrt{(q+1)^{2}-4\left(q^{2}+1+1\right)}\right] / 2 \\
& \text { and } \rho=(-1 \pm 2 i \sqrt{2}) / 3, \tau=-1 \pm i \sqrt{2}, \theta= \pm i \sqrt{2} .
\end{aligned}
$$

For the metric tensor $g_{i j}$ the $Z$ - and $H$-/E-eigendata are the common eigendata of square symmetric matrices, and are given by the equations,

$$
g_{i j} f^{j}=\lambda f^{i} \Leftrightarrow \frac{F^{2}}{n y^{i}}\left(\frac{2}{n} g *-g^{i}\right)
$$

Since $[g]$ is real and symmetric, it is diagonalizable, its eigenvalues are all real, the eigenspaces are mutually orthogonal, and its signature is $(+,-, \ldots,-)$. The matrix admits an orthonormal basis made of eigenvectors. Then for $n=2$, we have $[g]=(1 / 2)\left[e_{2}, e_{1}\right]$, the spectral data are

$$
S_{\lambda= \pm 1}=\{(1, \pm 1)\}^{\sigma}
$$

and $\mathrm{g}$ admits two distinct Candecomp rank-I approximations,

$$
g^{\sigma} m \pm \frac{1}{2}\left(e_{1} \pm e_{2}\right) \otimes\left(e_{1} \pm e_{2}\right)
$$

For $n=3$ and $n=4$, the matrix of the fundamental tensor is given by 


$$
\begin{aligned}
& {[g]_{\mathcal{H}_{3}}=\frac{1}{9(\mathrm{abc})^{2 / 3}}\left(\begin{array}{ccc}
-a^{2} & 2 a b & 2 a b \\
2 b a & -b^{2} & 2 b c \\
2 c a & 2 c b & -c^{2}
\end{array}\right)} \\
& {[g]_{\mathcal{H}_{4}}=\frac{1}{8(\mathrm{abcB})^{1 / 2}}\left(\begin{array}{cccc}
-a^{2} & a b & a c & a d \\
b a & -b^{2} & b c & b d \\
c a & c b & -c^{2} & c d \\
d a & d b & d c & -d^{2}
\end{array}\right)} \\
& \text { where }(a, b, c)=\left(\frac{1}{y^{1}}, \frac{1}{y^{2}}, \frac{1}{y^{3}}\right) \text { and respectively } \\
& (a, b, c, d)=\left(\frac{1}{y^{1}}, \frac{1}{y^{2}}, \frac{1}{y^{3}}, \frac{1}{y^{4}}\right) \text {. }
\end{aligned}
$$

Besides the applications in Relativity of the Berwald-Moor, Chernov and Bogoslovsky mth root models which presented above, similar models have been investigated as well by P.L. Antonelli ([1]) and by a group of scientists from University of Eindhoven, both focusing on the behavior of the associated Finslerian geodesics. An anisotropic metric, introduced to model General relativity was proposed in 1992 by Roxburgh ([34]):

$$
\begin{gathered}
F(x ; y)=\left[\mathrm{a}(\rho) \cdot\left(\mathrm{y}^{1}\right)^{4}+b(\rho) \cdot\left(\mathrm{y}^{1}\right)^{2}\left(\left(y^{3}\right)^{2}+\left(y^{4}\right)^{2}\right)^{2}+\right. \\
\left.+c(\rho) \cdot\left(\left(\mathrm{y}^{2}\right)^{2}+\left(y^{3}\right)^{2}+\left(y^{4}\right)^{2}\right)^{2}\right]^{1 / 4} \\
\text { where } x=\left(x^{1}, x^{2}, x^{3}, x^{4}\right), \text { and } \rho=\sqrt{\left(x^{2}\right)^{2}+\left(x^{3}\right)^{2}+\left(x^{4}\right)^{2}}
\end{gathered}
$$

A relatively recent Finslerian model was proposed by Bogoslovsky and Goenner ([15], [23]), whose spectral theory is subject of present and further research.

\section{Conclusions}

The spectral theory associated to several types of Finsler metrics, all of $m$-th root type was described. The (covariant) metric tensor fields, the Cartan tensors and the fundamental $m$-th root 
tensors were investigated: the spectral data were determined, and the outcoming impact of the results on the indicatrix theory of the models and on the related spectral decompositions was pointed out. Further research have in view insight on several alternative models, provided by the Finsler-type Bogoslovsky-Goenner, Randers and Shen structures.

\section{References}

1. Antonelli P.L., Ingarden R.S., Matsumoto M. (1991). The Theory of Spray and Finsler Spaces with Applications in Physics and Biology, Kluwer Acad. Publishers.

2. Astola L., Florack L. (2009). Finsler Geometry on higher order tensor fields and applications to high angular resolution diffusion imaging, LNCS 5567, 224-234.

3. Balan V. (2012). Spectra of multilinear forms associated to notable m-root relativistic models. Linear Algebra and Appl. (LAA), 436, 1, 152-162.

4. Balan V. (2012). Spectral data of Cartan and metric tensors in Berwald-Moor Finsler spaces, Rev. Roum. Math. Pures Appl., LVII, 35-42.

5. Balan V. (2010) Numerical multilinear algebra of symmetric m-root structures. Spectral properties and applications. In Symmetry: Culture and Science, Part 2; Geometric Approaches to Symmetry 2010, Budapest, Hungary, 21, 119-131.

6. Balan V. (2008). Spectral properties and applications of numerical multilinear algebra of m-th root structures. Hypercomplex Numbers in Geom. Phys., 2 (10), 101-107.

7. Balan V., Brinzei N. (2006). Einstein equations for (h,v)-Berwald-Moor relativistic models. Balkan J. Geom. Appl, 11, 2 , 20-26.

8. Balan V., Lebedev S. (2010) On the Legendre transform and Hamiltonian formalism in Berwald-Moor geometry. Diff. Geom. Dyn. Syst., 12, 4-11.

9. Balan V., Nicola I. R. (2009). Berwald-Moor metrics and structural stability of conformallydeformed geodesic SODE. Appl. Sci (APPS), 11, 19-34.

10. Balan V., Perminov N., Applications of resultants in the spectral m-th root framework. Appl. Sci. (APPS), 12, 20-29.

11. Bejancu A. (1990). Finsler Geometry and Applications. E.Horwood.

12. Bienvenu G., Kopp L. (1983). Optimality of high-resolution array processing using the eigensystem approach. IEEE Trans. ASSP 31, 1235-1248.

13. Brinzei N., Siparov S. (2008). The equations of electromagnetism in some special anisotropic spaces. Hypercomplex Numbers in Geometry and Physics, Ed.’Mozet”, Russia, 2 (10), 5, 44 55. 
14. Bogoslovsky G.Yu. (2008). Rapidities and observable 3-velocities in the flat Finslerian event space with entirely broken 3D isotropy? Symmetry, Integrability and Geometry, Methods and Applications, SIGMA 4, 045, 1-21.

15. Bogoslovsky G.Yu., Goenner H.F. (1998). On a possibility of phase transitions in the geometric structure of space-time. arXiv, gr-qc/9804082v1.

16. Cardoso J.F. (1999). High-order contrasts for independent component analysis. Neural Computation 11, 157-192.

17. Chang K.C., Pearson K., Zhang T. (2009). On eigenvalue problems of real symmetric tensors. Journal of Mathematical Analysis and Applications, 350, 416-422.

18. Chang Z., Li X. Modified Newton's gravity in Finsler Space as a possible alternative to dark matter hypothesis. arXiv, 0806.2184v2 [gr-qc].

19. Chernov V.M. (2007). On defining equations for the elements of associative and commutative algebras and on associated metric forms. Space-Time Structure. Algebra and Geometry, Lilia Print, Moscow, 189-209.

21. Comon P. (2000). Block methods for channel identification and source separation. IEEE Symposium on Adaptive Systems for Signal Process, Commun. Control, Canada, 87-92.

22. Comon P. (1994). Tensor diagonalization, a useful tool in signal processing. IFAC-SYSID, 10th IFAC Symposium on System Identification, 77-82.

23. Coppi R., Bolasco S. (1989). Multiway Data Analysis, Elsevier, Amsterdam, 1989.

24. Goenner H.F., Bogoslovsky G.Yu. (1996). A class of anisotropic (Finsler-) space-time geometries. Preprint NPI MSU 96, 35, 442.

25. Kofidis E., Regalia P.A. (2001). Tensor approximation and signal processing applications. Structured Matrices in Mathematics, Computer Science and Engineering, vol. I, Contemporary Mathematics 280, AMS, Providence.

26. de Lathauwer L. (2004). First-order perturbation analysis of the best rank-(R1;R2;R3) approximation in multilinear algebra. J. Chemometrics, 18, 2-11.

27. de Lathauwer L., de Moor B., Vandewalle J. (2000). A multilinear singular value decomposition. SIAM J. Matrix Anal. Appl., 21, 1253-1278.

28. Miron R., Anastasiei M. (1994). The Geometry of Vector Bundles. Theory and Applications. FTPH, no. 59, Kluwer Acad. Publishers.

29. Pavlov D.G. (2004). Four-dimensional time. Hypercomplex Numbers in Geom. Phys., 1 (1), 31-39.

30. Pavlov D.G. (2004). Generalization of scalar product axioms. Hypercomplex Numbers in 
Geom. Phys., 1 (1), 5-18.

31. Pavlov D.G., Kokarev S.S. (2008). Konformnye kalibrovki geometrii Berval'da-Moora i inducirovannye imi nelinejnye simmetrii [Conformal gauges of the Berwald-Moor Geometry and their induced non-linear symmetries]. Hypercomplex Numbers in Geom. Phys. 2 (10), 5, $3-14$.

32. Qi L. (2005). Eigenvalues of a real supersymmetric tensor. Jour. Symb. Comp., 40, 1302-1324.

33. Qi L. (2006). Rank and eigenvalues of a supersymmetric tensor, the multivariate homogeneous polynomial and the algebraic hypersurface it defines. Jour. Symb. Comp., 41, 1309-1327.

34. Qi L., Sun W., Wang Y. (2007). Numerical multilinear algebra and its applications. Front. Math. China, 2 (4), 501-526.

35. Roxburgh I.W. (1992). Post Newtonian tests of quartic metric theories of gravity. Rep. Math. Phys., 31, 171-178.

36. Vorobyov S.A., Rong Y., Sidiropoulos N.D., Gershman A.B. (2005). Robust iterative fitting of multilinear models. IEEE Trans. on Signal Processing, 53, 2678-2689.

37. Zarypov R.G. (2008). Model' fizicheskogo polja v sobstvennom trehmernom prostranstve dlja geometrii sobytij berval'da-moora [Model of the physical field in proper 3-dimensional space for the Berwald-Moor Geometry of events]. Hypercomplex Numbers in Geom. Phys., 2 (10), 5, 124-130. 